\title{
Probabilistic alignment leads to improved accuracy and read coverage for bisulfite sequencing data
}

\author{
Changjin Hong ${ }^{1}$, Nathan L Clement ${ }^{2}$, Spencer Clement ${ }^{3}$, Saher Sue Hammoud ${ }^{4,5}$, Douglas T \\ Carrell $^{4}$, Bradley R Cairns ${ }^{5}$, Quinn Snell ${ }^{3}$, Mark J Clement ${ }^{3}$ and William Evan Johnson ${ }^{* *}$
}

\begin{abstract}
Background: DNA methylation has been linked to many important biological phenomena. Researchers have recently begun to sequence bisulfite treated DNA to determine its pattern of methylation. However, sequencing reads from bisulfite-converted DNA can vary significantly from the reference genome because of incomplete bisulfite conversion, genome variation, sequencing errors, and poor quality bases. Therefore, it is often difficult to align reads to the correct locations in the reference genome. Furthermore, bisulfite sequencing experiments have the additional complexity of having to estimate the DNA methylation levels within the sample.

Results: Here, we present a highly accurate probabilistic algorithm, which is an extension of the Genomic Next-generation Universal MAPper to accommodate bisulfite sequencing data (GNUMAP-bs), that addresses the computational problems associated with aligning bisulfite sequencing data to a reference genome. GNUMAP-bs integrates uncertainty from read and mapping qualities to help resolve the difference between poor quality bases and the ambiguity inherent in bisulfite conversion. We tested GNUMAP-bs and other commonly-used bisulfite alignment methods using both simulated and real bisulfite reads and found that GNUMAP-bs and other dynamic programming methods were more accurate than the more heuristic methods.

Conclusions: The GNUMAP-bs aligner is a highly accurate alignment approach for processing the data from bisulfite sequencing experiments. The GNUMAP-bs algorithm is freely available for download at: http://dna.cs.byu.edu/ gnumap. The software runs on multiple threads and multiple processors to increase the alignment speed.
\end{abstract}

Keywords: DNA methylation, Bisulfite sequencing, Probabilistic alignment, Parallel processing

\section{Background}

DNA methylation is a chemical process in which a methyl group is added to the carbon-5 position of a DNA cytosine. In most vertebrates, DNA methylation typically occurs on the cytosine of a CpG dinucleotide $[1,2]$, although some specific examples of other types of methylation have been shown to play roles in specific tissues [3-6]. Since its discovery over 60 years ago, DNA methylation has been linked to many important biological phenomena such as the suppression of gene expression

\footnotetext{
*Correspondence: wej@bu.edu

${ }^{1}$ Division of Computational Biomedicine, Boston University School of Medicine, Boston, MA, USA

Full list of author information is available at the end of the article
}

[7,8], imprinting [9], X chromosome inactivation [10], epigenetic reprogramming during mammalian development [11], and cancer development [12]. Therefore, the study of genome-wide methylation patterns is currently of great interest to researchers, particularly in areas related to the molecular mechanisms of development, cancer, and chromatin dynamics.

When DNA is treated with sodium bisulfite, unmethylated cytosine residues are converted to uracil, while 5methylcytosine residues are unaffected. Later in bisulfite sequencing (BS-seq) experimental protocols, PCR amplification or sequencing converts the uracil residues to thymines. The next step of finding the correct genomic 
location for a bisulfite-treated read (BSR) is a complicated and difficult process. Although cytosine to thymine changes are allowed for when mapping the BSRs to the genomic sequence, methylated and unmethylated $\mathrm{CpG}$ locations are often identified and, in some cases, it is impossible to distinguish between a bisulfite (BS)-treated thymine that originated from an unmethylated cytosine, and a true thymine from a different genomic location or an individual genomic variation at that location $[13,14]$. This ambiguity is often magnified by the presence of sequencing errors or low-quality bases. As a result, sophisticated computational strategies are required for aligning reads from BS-seq experiments.

The first whole-genome methylation profiles were performed on Arabidopsis thaliana. To map the resultant BSRs, alignment algorithms based on a probabilistic formulation and a suffix tree [15] as well as reference genome conversion [16] were used. However, it is not computationally feasible to apply this approach to larger genomes such as the human genome, or to experiments with the current deeper sequencing depths. Later algorithms, such as BSMAP [14], constructed seed tables of locations from both the original reference sequence and the BS variants, and then extends the seeds to form a possible mapping location. This seed extension process can be somewhat unreliable because the seeds must be exact and do not take into account BS-treated variations. BS alignment methods such as BS Seeker [17], Bismark [18], and BRAT$B W$ [19] have been used to map BSRs. These methods employ a Burrows-Wheeler transformation [20] for fast (in)exact string matching and then combine the results with either a pre-processing or post-processing script to handle BSRs with three letters after converting all Cs to $\mathrm{T}$. The strength of these methods lie with reads with fewer mismatches, but they offer very limited support for aligning reads with insertions or deletions (indels). These methods also have difficulty in aligning Ts in the reads to $\mathrm{Cs}$ in the genome without also (incorrectly) aligning for cysteines in the reads to thymidines in the genome. More recently, the LAST alignment program has been adapted to align BSRs [21]. LAST uses a seed extension approach similar to the one used by NCBI BLAST [22], but the speed and mapping accuracy are increased by using variable-length seeds and base quality information [23].

In this study, we present a highly accurate probabilistic mapping algorithm, Genomic Next-generation Universal MAPper for Bisulfite Sequencing (GNUMAP$b s)$, for BSR alignment. GNUMAP-bs is an extension of the Genomic Next-Generation Universal MAPper (GNUMAP) [24] that can accommodate the alignment of BSRs to a reference genome. GNUMAP-bs was developed to achieve higher accuracy than other BS-seq approaches by including base quality scores in the alignment pro- cess. In addition, the GNUMAP-bs probabilistic mapping approach allows for the unbiased estimation of DNA methylation, especially when reads are aligned to multiple genomic locations.

\section{Results and discussion}

We compared GNUMAP- $b s$ with several commonly-used BS alignment methods, namely BSMAP (v2.3) [14], Bismark(v0.7.9) [18], LAST [21], and BRAT-BW [19]. We also compared GNUMAP-bs with the unpublished proprietary probabilistic aligner Novoalign (v2.07.09, http://www. novocraft.com) because it uses a probabilistic approach that is similar to the approach used in GNUMAP-bs. For a fair comparison, we considered only common options that were shared by all the alignment methods; otherwise, we used the default values or slight modifications of the defaults to achieve the best performance for each method. For each aligner, we allowed up to three or four base pair differences within 100-bp BSRs. For GNUMAP$b s$ and Novoalign, this resulted in cutoff values of -a 0.90 and $-t 75$, respectively. Considering that repeated genomic sequences often appear in mammalian genomes, we allowed up to 20 multiple mapping locations for each read. This approach to handling multiple alignments has only a small impact on the sensitivity of the more heuristic algorithms, but has moderate sensitivity gains in more traditional approaches like GNUMAP-bs. The Bismark software only reports up to two valid alignments. The parameter values that were used for each alignment method are given in Table 1.

We used two datasets to evaluate the performance of each of the alignment methods. The first was a simulated BSR dataset, which was carefully designed to mimic a typical human methylation experiment. The second was a real human BS-seq dataset, which was compared with an experimentally-derived human methylome profile.

\section{Table 1 Parameters used in the two experiments for each of the aligners tested}

\begin{tabular}{|c|c|}
\hline Mapper & Parameters \\
\hline GNUMAP-bs & -m 17 -s 1 - T 20 -a $0.90(-a$ 0.92) -b \\
\hline Novoalign & $-k 17-s 2-r-A 20-t 75(-t 90)-b 2$ \\
\hline$B S M A P$ & -n $0-w 100-v 3$ \\
\hline Bismark & $-n 2-150$ \\
\hline Bismark-bt2 & -N 1 -L 20 -bowtie2 -min-score L,0,-0.6 \\
\hline LAST & -Q1 -j1 -d120 -n20 -f1 | last-map-probs.py -s150 -m0.95 \\
\hline BRAT-BW & default settings for single-end reads \\
\hline
\end{tabular}

Parameters in parentheses were used for the human BSR dataset. Up to 3 to 4 sequencing errors or mutations were allowed in a 100-bp long BSR. Because the human genome contains many repeated genomic regions, 20 valid mapping locations were allowed for a given read when the alignment method supported this feature. 


\section{Simulated bisulfite sequencing experiment}

For the simulation study, we randomly assigned $20 \%$ of the CGs in the whole human genome (NCBI build37/HG19) to represent unmethylated cytosines (Cs) by changing them to thymidines (Ts), thereby simulating complete BS conversion. For the remaining $80 \%$ of the CGs, $75 \%$ was randomly assigned to be fully methylated and, therefore the Cs remained as Cs. The remaining 5\% was assigned to be methylated in proportions between 0.1 and 0.9 . In this dataset, we assumed that all non-CG sites remained unmethylated, so these Cs were all changed to Ts for read generation. We used the $d w g s i m$ (http://sourceforge.net/ projects/dnaa/files/dwgsim) simulation tool with parameters -e 0.001-0.008 -1 100 -y 0.05 -r 0.002 -R 0.2 -C 10, to generate 100-bp BSRs with a 10 ? read depth across the genome. This simulation produced a BS-seq dataset that contained approximately 180 million (M) reads with a sequencing error rate that ranged from 0.001 to 0.008 and increased from the 5 ' to 3 ' ends, plus $5 \%$ randomly generated sequence, and a mutation rate of 0.002 (in which $0.2 \%$ were indels).

We found that the mapping sensitivities of the probabilistic aligners GNUMAP-bs (97.0\%), Novoalign (96.3\%), and LAST (96.9\%), were higher than the sensitivities of BSMAP (93.9\%) and Bismark (93.2\%), as shown in Table 2. The Bismark-Bowtie 2 algorithm had the lowest false positive rate $(0.1 \%)$, but at the cost of an approximately $7 \%$ decrease in its sensitivity. Overall, the Bismark-Bowtie 2 algorithm had 1.2 M fewer false positives than GNUMAP$b s$, but approximately $10 \mathrm{M}$ fewer BSRs were aligned correctly to the genome. The Bismark-Bowtie algorithm performed better than Bismark-Bowtie2 in that it aligned $5 \mathrm{M}$ more reads with only a slight increase in the number of incorrectly aligned reads. The $L A S T$ algorithm aligned nearly all the BSRs (99.2\%) to the genome, but although the proportion of correctly mapped reads was similar to that of GNUMAP-bs, the error rate was much higher. $B R A T-B W$ was not included in this comparison because the application software removed the original sequence header/name which contained information pointing to the correct alignment location of the BSRs.

Differences in sensitivities of these methods were much more pronounced for BSRs that contained at least one sequencing error or genome variant, and the GNUMAP$b s$ sensitivity was clearly better than the sensitivities of the other approaches (Table 2). We also evaluated how accurately each aligner predicted the CG methylation levels when the true methylation level ranged from $10 \%$ to $90 \%$. All the alignment methods tested performed well with mean absolute errors less than 0.01 and with standard deviations less than 0.07 .

When the computational performances of each of the methods were compared (Table 2), we observed that GNUMAP-bs required the most RAM $(44.8 \mathrm{~GB})$ and Bismark required the least (5.9 GB). Because some of the software applications presented here support

Table 2 Simulated bisulfite read experiment

\begin{tabular}{|c|c|c|c|c|c|c|}
\hline Evaluation metric & GNUMAP-bs & Novoalign & $B S M A P$ & Bismark & Bismark-bt2 & LAST \\
\hline \multicolumn{7}{|l|}{ Overall mapping results: } \\
\hline Total reads aligned (\%) & 156.6M(97.8) & 155.8M(97.4) & 153.4M(95.9) & $149.5 \mathrm{M}(93.4)$ & $145.4 \mathrm{M}(90.8)$ & $158.7 \mathrm{M}(99.2)$ \\
\hline Correctly aligned (\%) & $155.2 \mathrm{M}(\mathbf{9 7 . 0})$ & 154.2M(96.3) & 150.2M(93.9) & 149.2M(93.2) & $145.2 \mathrm{M}(90.7)$ & $155.1 \mathrm{M}(96.9)$ \\
\hline Incorrectly aligned (\%) & $1.4 \mathrm{M}(0.9)$ & $1.7 \mathrm{M}(1.1)$ & $1.5 \mathrm{M}(1.0)$ & $0.3 \mathrm{M}(0.2)$ & $0.2 \mathrm{M}(\mathbf{0 . 1})$ & $3.6 \mathrm{M}(2.3)$ \\
\hline \multicolumn{7}{|l|}{ With $\geq 1$ sequence variant: } \\
\hline Total reads aligned (\%) & $69.0 M(97.8)$ & 66.0M(93.6) & $65.3 \mathrm{M}(92.6)$ & $63.6 \mathrm{M}(90.2)$ & $59.6 \mathrm{M}(84.4)$ & $70.3 \mathrm{M}(\mathbf{( 9 9 . 7 )}$ \\
\hline Correctly aligned (\%) & $67.7 \mathrm{M}(\mathbf{9 6 . 0})$ & $65.3 \mathrm{M}(92.6)$ & $63.9 \mathrm{M}(90.1)$ & $63.3 \mathrm{M}(89.8)$ & $59.4 M(84.1)$ & $66.7 \mathrm{M}(94.6)$ \\
\hline Incorrectly aligned (\%) & $1.3 \mathrm{M}(1.8)$ & $0.7 M(1.0)$ & $1.4 M(2.0)$ & $0.3 \mathrm{M}(0.4)$ & $0.2 \mathrm{M}(\mathbf{0 . 3})$ & $3.5 \mathrm{M}(5.1)$ \\
\hline \multicolumn{7}{|l|}{ Predicted methylation: } \\
\hline Ave. absolute estimation err. & 0.11 & 0.69 & 0.22 & 0.11 & 0.10 & - \\
\hline Standard err. & 0.056 & 0.066 & 0.067 & 0.064 & 0.062 & - \\
\hline \multicolumn{7}{|l|}{ Computational resource: } \\
\hline Total compute time (16 CPUs) & 39 h 50 m & $29 \mathrm{~h} 25 \mathrm{~m}$ & $4 \mathrm{~h} 28 \mathrm{~m}$ & $46 \mathrm{~h} 16 \mathrm{~m}$ & $97 \mathrm{~h} 26 \mathrm{~m}$ & $58 \mathrm{~h} 20 \mathrm{~m}$ \\
\hline Peak memory usage (GB) & 44.8 & 14.5 & 9.4 & 5.9 & 7.9 & 15.9 \\
\hline Reads per second per CPU & 68 & 92 & 607 & 448 & 26 & 753 \\
\hline
\end{tabular}

Simulation study of 160 million (M) simulated BSRs generated from the human genome reference sequence. The GNUMAP-bs algorithm was the most sensitive aligner, especially for reads with $\geq 1$ sequence variant (sequencing errors or mutations). The Bismark algorithm had the smallest error rate with $1.2 \mathrm{M}$ fewer erroneously assigned reads than GNUMAP-bs, however GNUMAP-bs correctly aligned 6 to $10 \mathrm{M}$ more reads. The BSMAP algorithm had the fastest total run time, however its sensitivity was less than the sensitivity of the GNUMAP-bs algorithm. LAST mapped nearly all reads with a sensitivity that was comparable to that of GNUMAP-bs, but the mapping error rate for LAST was much higher than it was for GNUMAP-bs. 
computation on multiple threads and some do not, we presented two different measures of computational speed: 1) the total run time on a $16 \mathrm{CPU}$ linux server and 2) the number of reads processed per CPU per second. GNUMAP-bs required approximately 40 hours of total compute time to process the $180 \mathrm{M}$ BSRs, while BSMAP, the fastest algorithm, was nearly nine times faster than GNUMAP-bs. The LAST application does not support parallel computing, so $L A S T$ had the longest total alignment time. However, the $L A S T$ algorithm aligned the most reads per second per CPU (753), while BismarkBowtie 2 aligned the least (26).

\section{Human BS-seq dataset}

We also evaluated the performances of the alignment methods using a BS-seq dataset generated from samples from a healthy human donor collected at the Andrology Laboratory at the University of Utah (Salt Lake City, UT, USA). The BS-seq data were generated by coupling BS conversion and the Illumina HiSeq2000 platform, which generated 101-bp BSRs for analysis. We aligned 283.6 M reads from three lanes of BS sequencing data containing $85 \mathrm{M}$ to $100 \mathrm{M}$ sequencing reads each, to the recent build of the human genome (NCBI build37/HG19). The human BSRs were processed for quality control as suggested previously [25]. Briefly, the quality control involved masking low quality bases or trimming consecutive lowest quality bases at the 3' ends of the reads.

We used the same parameters on these data as were used for the simulation experiment (Table 1) with two exceptions: -a 0.92 for GNUMAP-bs and -t 90 for Novoalign. The LAST algorithm again aligned the highest proportion (93.7\%) of BSRs to the genome, followed by $70.0 \%$ for GNUMAP-bs, $68.2 \%$ for Novoalign, $67.1 \%$ for BSMAP, 67.0\% for Bismark, 62.9\% for Bismark-Bowtie2, and only $50.3 \%$ for $B R A T-B W$.

We compared these mapping results with the Sangerbased BS sequencing control data available from the Human Epigenome Project (HEP) [26] by selecting the data that were obtained using the same type of tissue as was used in our BS-seq dataset. The HEP data provides a natural gold standard for algorithmic evaluation. For this comparison, we focused on the 13,563 HEP CG sites on chromosome 22 (chr22), because these data provide the most comprehensive chromosomal CG coverage that is available in the HEP database. The data for the other chromosomes in the HEP showed similar profiles as chr22, but the coverage was much sparser.

Overall, the mapping results produced by the different alignment methods for the human BS-seq dataset were consistent with the results for the simulation dataset. Compared with GNUMAP- $b s, L A S T$ aligned almost twice as many reads to chr22 (3.02 M compare with $1.57 \mathrm{M})$; however, the CG read coverage for LAST (7.6 reads/CG) was only about $20 \%$ higher than for GNUMAP-bs $(6.3$ reads/CG) (Table 3). While Novoalign aligned more reads to and covered more CG sites on chr22 than GNUMAP-bs, GNUMAP-bs had better CG read coverage $(6.3$ reads/CG) than Novoalign (6.1 reads/CG) and the other aligners, BSMAP (5.8 reads/CG), Bismark (5.8 reads/CG), Bismark-Bowtie2 (5.7 reads/CG), and BRAT$B W(5.0$ reads/CG) (Table 3$)$.

The differences between GNUMAP-bs and LAST become even less pronounced when the overlap between the mapped BSRs and the 13,563 CGs with HEP coverage on chr22 was considered. Last aligned the BSRs to $63.5 \%(8,606)$ of the CGs HEP sites compared with $58.3 \%$ $(7,902)$ for GNUMAP-bs, and $57.5 \%(7,802)$ for Novoalign, $57.1 \%(7,747)$ for BSMAP, 55.7\% (7,561) for Bismark, $54.1 \%(7,331)$ for Bismark-Bowtie 2 , and $49.3 \%(6,690)$ for $B R A T-B W$ (Table 3).

To determine if increased coverage resulted in decreased alignment quality, we computed the correlation coefficient between the estimated HEP methylation levels and the methylation levels estimated from each alignment method. The correlation coefficients were 0.887 for GNUMAP-bs and ranged from 0.895 for BismarkBowtie 2 to 0.882 for LAST (Table 3). We also computed a concordance statistic for each of the methods as defined previously [27]. Briefly, the concordance is the fraction of sites for which the methylation levels (aligner vs. HEP) differ by less than a predefined cutoff (we used 0.25). Based on this statistic, we found that the performances of most of the aligners were similar, with concordance values of 0.869 for GNUMAP-bs and ranged from 0.872 for Bismark-Bowtie2 0.872 and BRAT-BW to 0.858 for LAST (Table 3). These results showed that the increased coverage levels produced by $L A S T$ result in lower consistency with the HEP data in terms of both correlation and concordance. Pairwise comparisons of the concordance for the CGs covered/not covered between GNUMAP-bs and the other aligners are shown in Figure 1. For example, Figure 1 shows 577 CGs that were covered by GNUMAP$b s$ but not covered by Bismark; the concordance of the GNUMAP-bs methylation estimates for these sites was 0.78. In contrast, only six CGs covered by Bismark were not covered by GNUMAP-bs, and the concordance for these sites was extremely low (0.33). These results clearly showed that the increased mapping coverage (for both numbers of CGs covered and reads per CG) obtained using GNUMAP-bs did not result in decreased alignment quality or methylation estimates.

\section{Conclusions}

BS sequencing presents difficult challenges to researchers attempting to process the sequencing reads from BSseq experiments. In this work, we present GNUMAP$b s$, a highly accurate and effective alignment algorithm 
Table 3 Human bisulfite read experiment

\begin{tabular}{|c|c|c|c|c|c|c|c|}
\hline Evaluation metric & GNUMAP-bs & Novoalign & $B S M A P$ & Bismark & Bismark-bt2 & LAST & BRAT-BW \\
\hline \multicolumn{8}{|l|}{ All chr22: } \\
\hline Reads Aligned & $1.57 \mathrm{M}$ & $1.65 \mathrm{M}$ & $1.34 \mathrm{M}$ & $1.31 \mathrm{M}$ & $1.21 \mathrm{M}$ & $3.02 \mathrm{M}$ & $0.98 \mathrm{M}$ \\
\hline CGs Covered & $330 \mathrm{~K}$ & $336 K$ & $330 \mathrm{~K}$ & $310 \mathrm{~K}$ & $299 K$ & $373 K$ & $275 K$ \\
\hline CG read coverage & 6.3 & 6.1 & 5.8 & 5.8 & 5.7 & 7.6 & 5.0 \\
\hline \multicolumn{8}{|l|}{ HEP overlap: } \\
\hline CGs Covered & 7,902 & 7,802 & 7,747 & 7,561 & 7,331 & 8,606 & 6,690 \\
\hline Correlation & 0.887 & 0.889 & 0.887 & 0.888 & 0.895 & 0.882 & 0.894 \\
\hline Concordance & 0.869 & 0.867 & 0.865 & 0.867 & 0.872 & 0.858 & 0.872 \\
\hline CG Read coverage & 4.6 & 4.5 & 4.4 & 4.3 & 4.3 & 4.9 & 3.7 \\
\hline
\end{tabular}

Study of 162.3 M Illumina BSRs on human chromosome 22 (chr22) from a human donor sample. The top rows show the overall mapping efficiency, including covered CGs sites and read coverage aligned to chr22. The bottom rows show the consistency of each mapping result with known methylation levels at 13,563 CGs sites on chr22 available from the HEP database. The concordance statistic is defined as the fraction of overlapped CGs covered by the aligner with HEP CGs for which the methylation prediction error was smaller than 0.25 .

that is specifically designed to estimate DNA methylation levels with base-level resolution in BS-seq data. GNUMAP-bs uses a probabilistic approach to align BSRs to a reference genome. GNUMAP-bs was developed to achieve higher coverage and accuracy than other published BS-seq approaches by integrating base quality and alignment quality information in the mapping process. We have shown that the GNUMAP-bs probabilistic mapping approach results in an improved unbiased estimation of DNA methylation across the human genome. In simulated and real datasets, we showed that GNUMAP$b s$ outperforms other BS-seq alignment methods when both coverage and consistency were balanced with Sanger based BS sequencing controls.

In addition, GNUMAP-bs provides many high-demand features needed for constructing a high quality methylome from BS-seq data. First, GNUMAP-bs incorporates quality sequencing data into a dynamic programming framework. This feature gives GNUMAP- $b s$ the best balance between sensitivity and specificity of the tested BSR aligners, especially for reads that contain short polymorphisms. Second, GNUMAP-bs adaptively assigns an optimal mapping stringency based on an effective read length after the original read is trimmed. Third, GNUMAP-bs

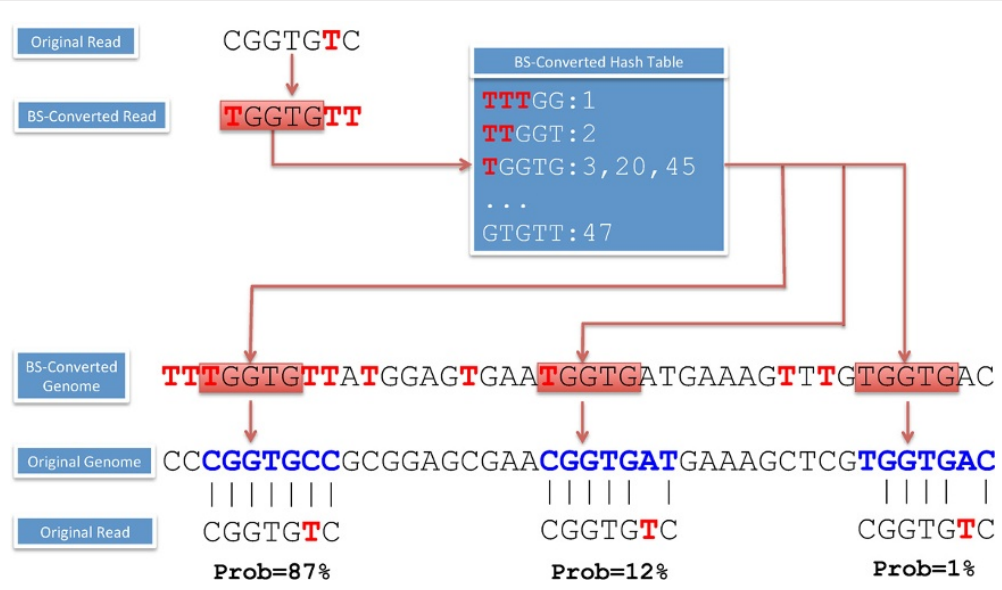

Figure 1 GNUMAP-bs workflow for mapping high-throughput bisulfite reads. A flow-chart of the GNUMAP-bs algorithm is shown. For the reference genome, all Cs are converted to Ts and then each $k$-mer in the genome is hashed, producing a list of positions in the genome to which the $k$-mer sequence is mapped. Given a completely BS-converted read (e.g. TGGTGTT) where the corresponding original bisulfite read (BSR) is known (e.g. CGGTGTC), a query k-mer (e.g. TGGTG) is searched against the BS-converted hash table. Locations with a k-mer match to both the genome and the read are aligned using the original read and original genome and the GNUMAP-bs probabilistic Needleman-Wunsch (N-W) algorithm. If the alignment score passes a quality threshold, the location is considered a match and recorded on the genome for future output. The posterior probability is computed for each mapped location using Equation (1). Finally, all the mapped BSRs are used to quantify the fraction of methylation at each CG location across the reference genome. 
not only relies on the maximal score alignment but also probabilistically considers suboptimal alignments; that is, the alignment score is converted to a posterior probability and the probabilistic scores quantify the likelihood of the true source location for each read across the reference genome. As a result, for both the simulated and real datasets, we showed that GNUMAP-bs was more effective that the other methods in detecting read locations in the presence of sequencing errors. GNUMAP-bs also displayed the highest consistency with a known HEP methylation database. Because GNUMAP-bs supports Message Passing Interface (MPI) processing, the computational burden of the dynamic programming can be alleviated. Moreover, with computer resources becoming cheaper, computing clusters with large numbers of nodes and cores, and more computing clouds are becoming available. Therefore, memory and CPU running times are less of a bottleneck, which is especially useful for GNUMAP$b s$ alignments. For this reason, accuracy should currently be a more important concern in BS-seq data analysis.

\section{Methods}

The GNUMAP-bs alignment algorithm is a modification of the GNUMAP algorithm, which consists of three main steps, all of which needed to be modified to align BSRs to a reference genome. A flow chart of the GNUMAP$b s$ algorithm is displayed in Figure 2. The first step is the construction of a hash table using all genomic subsequences, where the $k$ nucleotide (nt) long ( $k$-mers) are the keys and the hash table values store the genomic locations of the $k$-mer. In addition, $k$-mers from the BSRs are incrementally referenced in the reads in the genomic hash table. In GNUMAP-bs, the genome and the reads are artificially 'BS-converted' by changing all Cs to Ts before the hashing step. This process ensures that all BSRs can be referenced into the hash table regardless of whether or not they contain methylated bases.

In the second step of the GNUMAP algorithm, all the BSRs are aligned to the genome at the hashed locations. The alignment is performed using a novel probabilistic alignment algorithm that uses base quality information. All the regions that meet a predefined alignment score threshold are retained for the final step. In GNUMAP-bs, the probabilistic alignment algorithm matches the unconverted (original) reads to the unconverted genome while allowing matches between $\mathrm{Ts}$ in the reads and $\mathrm{Cs}$ in the genome. Using dynamic programming in probability space for mapping BSRs to a genome has several benefits: 1) the Needleman-Wunsch algorithm is guaranteed to find the optimal alignment for a BSR; 2) by incorporating base qualities into the probabilistic algorithm, true DNA methylation can be more accurately identified in the alignment, especially in areas where the reads have low base quality; and 3) by making only a small change in the alignment scoring matrix, namely by removing the $\mathrm{T}$ (read) to $\mathrm{C}$ (genome) 'mismatch' penalty and scoring these alignments as a 'match', the probabilistic Needleman-Wunsch algorithm can accurately account for the BS changes in BSRs without losing reads that are partially converted, or that have genome variations or sequencing errors.

During PCR amplification after BS conversion, the $\mathrm{C}$ to $\mathrm{T}$ conversions in the BSRs lead to $\mathrm{G}$ to A conversions

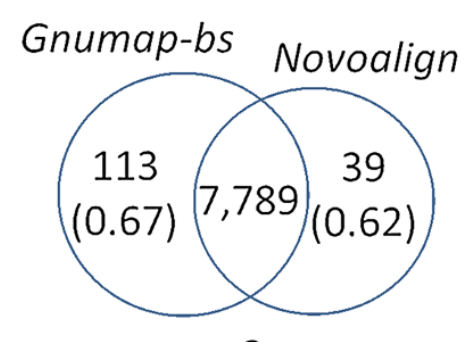

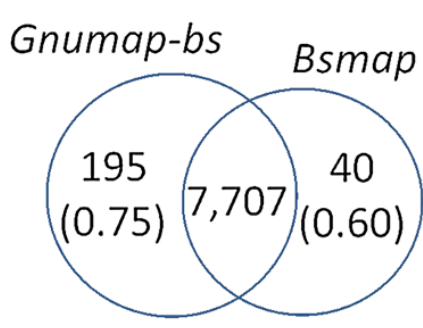

b

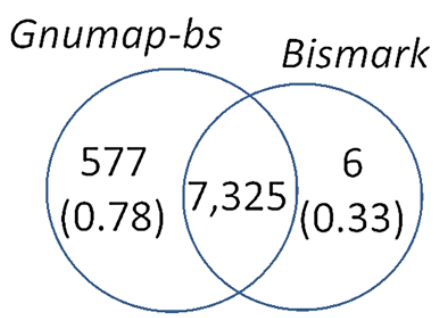

C

Figure 2 Relative complement mapping consistency of GNUMAP-bs with HEP methylation profiles of human chromosome 22. Venn diagrams between GNUMAP-bs and (a) Novoalign, (b) BSMAP, and (c) Bismark showing both the number of covered/uncovered CG sites and the concordance (in parenthesis) of these sites with the HEP methylation profiles. The estimated levels of methylation in the additional CG sites covered by the probabilistic aligners but not by the other aligners are highly concordant with the HEP results. 
on the PCR-synthesized strand as described previously [14]. These $G$ to A changes provide evidence of nonmethylation on the original strand. The phenomenon also occurs for $\mathrm{C}$ to $\mathrm{T}$ changes on the PCR-synthesized strand. Therefore, the GNUMAP-bs algorithm maps each BSR twice, once looking for only $\mathrm{C}$ to $\mathrm{T}$ changes and then looking for only $G$ to $A$ changes. When a $G$ to $A$ change is observed, the read is considered to be a PCR-synthesized strand, and the non-methylation event is attributed to the original strand.

In the final step, the GNUMAP-bs algorithm quantifies the significance of an alignment (e.g. a posterior probability) by assigning a mapping score that is proportional to the relative quality of alignments and inversely proportional to the number of matching sites in the reference genome. Given a BSR, $r$, assume that there are $J$ "best match" locations (potential mapping locations) for which the alignment score is $q[d]$ for alignment to genomic position $d$, which is also aligned to the first base pair location of $r$, matches some user-defined threshold. Then, the posterior probability at each location $P\left(r_{d}\right)$ can be computed as:

$$
P\left(r_{d}\right)=\frac{e^{q[d]}}{\sum_{j=1}^{J} e^{q[j]}}
$$

It is worth noting that the calibrated posterior probability is a special case of the LAST [21] mapping probabilities equation where a scaling factor for a bit score in the simple E-value statistics is not explicitly computed.

This probability provides an intuitive value between 0 and 1 representing the methylation signal for each read at each CG site. Other BS mapping programs use $\frac{1}{\text { \#mappings }}$ instead of Equation (1), which may not accurately capture the true posterior probability. The posterior probability indicates the relative significance of mapping a read to a particular position. The probabilistic NeedlemanWunsch computes a log-likelihood score; therefore, if a particular BSR has multiple possible mapping locations in the reference genome, the significance of each mapping decreases. After GNUMAP-bs computes $P\left(r_{d}\right)$ for each read, the algorithm combines the probabilities into one methylation profile vector and infers a true methylation ratio at each CG site.

For example, consider a nucleotide, $n_{i}$ at genomic location $i$, and assume that $n_{i}$ is a $C$ residue in a reference genome. Let $C^{(m)}$ be a methylated $C$ residue and $L(r)$ be the read length. For a set of aligned reads covering $i$, the methylation ratio $c[i]$ can be computed as:

$$
c[i]=\frac{\sum P\left(r_{d} \mid n_{i}=C^{(m)}\right)}{\sum P\left(r_{d}\right)}, d \leq i \leq d+L\left(r_{d}\right)
$$

Intuitively, the reads that cover each $C$ location across the reference genome provide evidence of true map- ping so that a reliable methylation percentage can be obtained.

\section{Software implementation and availability}

The GNUMAP-bs algorithm is integrated into the is GNUMAP software suite, and is freely available for download at http://dna.cs.byu.edu/gnumap. The GNUMAP-bs pipeline can accommodate single-end or pair-end reads in either FASTA or FASTQ file formats. A reference genome file (or multiple reference files) in FASTA format is also required. However, to increase the efficiency of the workflow, users can opt to write the reference genome hash table to a file, which can be used in future runs. GNUMAP outputs read alignments in standard SAM file format. The GNUMAP-bs software suite contains an addition application function (sam2gmp) that summarizes a SAM file and writes it into a text file that contains one line for each cytosine in the genome. Each line in this file contains the chromosome number, the location of the cytosine on the chromosome, the number of reads, and the numbers of As, Cs, Gs, Ts, and Ns covering the location. In addition, the text file gives a likelihood ratio p-value that indicates whether there is a significant number of $\mathrm{Cs}$ at the location (i.e. methylation significance).

In addition to the adaptations for BSRs, the GNUMAPbs software also contains several modifications to reduce the computational time and memory needed for the alignments. For example, the initial read and genome conversion step reduces the genome alphabet to three bases, leading to an increase in the number of genome locations identified in the hash table. To reduce this effect, multiple seeds from each read are referenced by GNUMAP-bs, and the alignment is only conducted on the locations with the top two most $k$-mer hash references. Furthermore, although the original GNUMAP software supports multi-threaded computing within the same node using pthread, GNUMAP-bs is fully enabled to support MPI processing. This feature allows a large-scale alignment to be spread across multiple nodes in a cluster or supercomputing facility [28]. In this implementation, the genome is divided into equal parts across nodes, and then the same batches of reads are aligned by each node to their individual portion of the genome. Once the batch is completed, the nodes communicate via MPI to calculate the posterior probability scores. Because most of the CPU time is spent on the alignments, the communication overhead is relatively small, resulting in a highly efficient parallel algorithm.

\footnotetext{
Competing interests

The authors declare that they have no competing interests.
}

Authors' contributions

QS, MJC, and WEJ conceived the study and developed the algorithm. $\mathrm{CH}$ and NLC implemented the algorithm and developed the software. $\mathrm{CH}$ and SC generated the simulated read dataset and analyzed both datasets. SSH, DTC, 
and BRC generated the human BS-seq datasets. $\mathrm{CH}$ and WEJ wrote the paper. All authors read and approved the final manuscript.

\section{Acknowledgements}

This research was conducted using the Fulton Supercomputing Lab at Brigham Young University (Provo, UT, USA) and the Linux Clusters for Genetic Analysis (LinGA) computing resources at Boston University Medical Campus (Boston, MA, USA). The study was supported financially by a grant from the National Institutes of Health (NIH) (R01 HG005692).

\section{Author details}

${ }^{1}$ Division of Computational Biomedicine, Boston University School of Medicine, Boston, MA, USA. ${ }^{2}$ Department of Computer Science, University of Texas, Austin, TX, USA. ${ }^{3}$ Department of Computer Science, Brigham Young University, Provo, UT, USA. ${ }^{4}$ IVF and Andrology Laboratories, Departments of Surgery, Obstetrics and Gynecology, and Physiology, University of Utah School of Medicine, Salt Lake City, UT, USA. ${ }^{5}$ Department of Oncological Sciences, Huntsman Cancer Institute, Salt Lake City, UT, USA

Received: 7 June 2013 Accepted: 19 November 2013

Published: 21 November 2013

\section{References}

1. Clark S, Harrison J, Frommer M, et al.: CpNpG methylation in mammalian cells. Nat Genet 1995, 10:20-27.

2. Bernstein BE, Meissner A, Lander ES: The mammalian epigenome. Cell 2007, 128(4):669-681.

3. Ramsahoye BH, Biniszkiewicz D, Lyko F, Clark V, Bird AP, Jaenisch R: Non-CpG methylation is prevalent in embryonic stem cells and may be mediated by DNA methyltransferase 3a. Proc Nat Acad Sci 2000 97(10):5237-5242. http://www.pnas.org/content/97/10/5237.abstract.

4. Haines TR, Rodenhiser DI, Ainsworth PJ: Allele-Specific non-CpG methylation of the $\mathrm{Nf1}$ Gene during early mouse development. Dev Biol 2001, 240(2):585-598. http://www.sciencedirect.com/science/ article/pii/S0012160601905044.

5. Grandjean V, Yaman R, Cuzin F, Rassoulzadegan M: Inheritance of an epigenetic mark: the CpG DNA Methyltransferase 1 is required for de novo establishment of a complex pattern of non-CpG methylation. PLOS ONE 2007, 2(11):e1136. http://www.plosone.org/ article/info\%3Adoi\%2F10.1371\%2Fjournal.pone.0001136.

6. Lister R, Pelizzola M, Dowen R, Hawkins R, Hon G, Tonti-Filippini J, Nery J Lee $L$, Ye Z, Ngo Q, et al.: Human DNA methylomes at base resolution show widespread epigenomic differences. Nature 2009, 462(7271):315-322

7. Riggs $A D: \mathbf{X}$ inactivation, differentiation, and DNA methylation. Cytogenet Cell Genet 1975, 14:9-25.

8. Holliday R, Pugh JE: DNA modification mechanisms and gene activity during development. Science 1975, 187(4173):226-232.

9. Morison IM, Ramsay JP, Spencer HG: A census of mammalian imprinting. Trends Genet 2005, 21(8):457-465.

10. Chow JC, Yen Z, Ziesche SM, Brown CJ: Silencing of the mammalian $\mathbf{X}$ chromosome. Annu Rev Genomics Hum Genet 2005, 6:69-92.

11. Reik W: Stability and flexibility of epigenetic gene regulation in mammalian development. Nature 2007, 447(7143):425-432

12. Feinberg AP, Vogelstein B: Hypomethylation distinguishes genes of some human cancers from their normal counterparts. Nature 1983, 301(5895):89-92.

13. Frommer M, McDonald L, Millar D, Collis C, Watt F, Grigg G, Molloy $P$, Paul C: A genomic sequencing protocol that yields a positive display of 5-methylcytosine residues in individual DNA strands. Proc Nat Acad Sci 1992, 89(5):1827.

14. Xi Y, Li W: BSMAP: whole genome bisulfite sequence MAPping program. BMC Bioinformatics 2009, 10:232

15. Cokus S, Feng S, Zhang X, Chen Z, Merriman B, Haudenschild C, Pradhan S, Nelson S, Pellegrini M, Jacobsen S: Shotgun bisulphite sequencing of the Arabidopsis genome reveals DNA methylation patterning. Nature 2008, 452(7184):215-219.

16. Lister R, O'Malley R, Tonti-Filippini J, Gregory B, Berry C, Millar A, Ecker J: Highly integrated single-base resolution maps of the epigenome in Arabidopsis. Cell 2008, 133(3):523-536.
17. Chen P, Cokus S, Pellegrini M: BS Seeker: precise mapping for bisulfite sequencing. BMC Bioinformatics 2010, 11:203. http://www. biomedcentral.com/1471-2105/11/203.

18. Krueger $F$, Andrews SR: Bismark: a flexible aligner and methylation caller for Bisulfite-Seq applications. Bioinformatics 2011, 27(11):1571-1572. http://bioinformatics.oxfordjournals.org/content/27/ 11/1571.long; http://dx.doi.org/10.1093/bioinformatics/btr167.

19. Harris EY, Ponts N, Roch KGL, Lonardi S: BRAT-BW: efficient and accurate mapping of bisulfite-treated reads. Bioinformatics 2012 28(13):1795-1796. http://bioinformatics.oxfordjournals.org/content/28/ 13/1795.long; http://dx.doi.org/10.1093/bioinformatics/bts264.

20. Burrows $M$, Wheeler D: A block-sorting lossless data compression algorithm. 1994, Technical Report SRC-RR-124, Digital Equipment Corporation, 1-16.

21. Frith $M C$, Mori $R$, Asai $K:$ A mostly traditional approach improves alignment of bisulfite-converted DNA. Nucleic Acids Res 2012, 40(13):e100-e100. http://nar.oxfordjournals.org/content/38/7/e96.short.

22. Altschul SF, Madden TL, Shaffer AA, Zhang J, Zhang Z, Miller W Lipman DJ: Gapped BLAST and PSI-BLAST: a new generation of protein database search programs. Nucleic Acids Res 1997, 25(17):3389-402

23. Frith $M C$, Wan $R$, Horton $P$ : Incorporating sequence quality data into alignment improves DNA read mapping. Nucleic Acids Res 2010 38(7):100-100. http://nar.oxfordjournals.org/content/38/7/e100.long.

24. Clement NL, Snell Q, Clement MJ, Hollenhorst PC, Purwar J, Graves BJ, Cairns BR, Johnson WE: The GNUMAP algorithm: unbiased probabilistic mapping of oligonucleotides from next-generation sequencing. Bioinformatics 2010, 26:38-45. http://bioinformatics. oxfordjournals.org/content/26/1/38; http://dx.doi.org/10.1093/ bioinformatics/btp614.

25. Chatterjee A, Stockwell P, Rodger E, Morison I: Comparison of alignment software for genome-wide bisulphite sequence data. Nucleic Acids Res 2012, 40(10):e79.

26. Eckhardt F, Lewin J, Cortese R, Rakyan V, Attwood J, Burger M, Burton J, Cox T, Davies R, Down T, et al.: DNA methylation profiling of human chromosomes 6, 20 and 22. Nat Genet 2006, 38(12):1378-1385.

27. Harris RA, Wang T, Coarfa C, Nagarajan RP, Hong C, Downey SL, Johnson BE, Fouse SD, Delaney A, Zhao Y, Olshen A, Ballinger T, Zhou X, Forsberg KJ, Gu J, Echipare L, O'Geen H, Lister R, Pelizzola M, Xi Y, Epstein CB, Bernstein BE, Hawkins RD, Ren B, Chung WY, Gu H, Bock C, Gnirke A, Zhang MQ, Haussler D, Ecker JR, Li W, Farnham PJ, Waterland RA, Meissner A, Marra MA, Hirst M, Milosavljevic A, Costello JF: Comparison of sequencing-based methods to profile DNA methylation and identification of monoallelic epigenetic modifications. Nat Biotechnol 2010, 28(10):1097-1105.

28. Clement N, Clement M, Snell Q, Johnson W: Parallel Mapping Approaches for GNUMAP. 2012 IEEE 26th Int Parallel Distributed Process Symp Workshops PhD Forum 2011, 0(1530-2075):435-443.

doi:10.1186/1471-2105-14-337

Cite this article as: Hong et al:: Probabilistic alignment leads to improved accuracy and read coverage for bisulfite sequencing data. BMC Bioinformat ics 2013 14:337. 